\title{
The bldA-encoded tRNA is poorly expressed in the bldI mutant of Streptomyces coelicolor A3(2)
}

\author{
Brenda K. Leskiw and Richard Mah
}

Department of Biological Sciences, University of Alberta, Edmonton, Alberta, Canada T6G 2E9
Author for correspondence: Brenda K. Leskiw.Tel: +1 403492 1868. Fax: +1 4034929234.

e-mail: brenda_leskiw@biology.ualberta.ca

\begin{abstract}
The bldA gene, encoding a leucyl tRNA recognizing the UUA codon, is expressed at significantly lower levels in the bldl mutant, Streptomyces coelicolor J703, than in the parent 5. coelicolor A3(2). Expression of a TTAcontaining reporter gene was reduced in the b/dl mutant, as was the mature, 87 nucleotide, form of the b/dA-encoded tRNA. This reduced level of the tRNA was also seen when the bldA gene was introduced on a high-copy-number plasmid into the bld/ mutant, suggesting that maximal b/dA expression may require a bldl-dependent promoter.
\end{abstract}

Keywords: Streptomyces coelicolor, bld $A$, bldI, antibiotic production, morphological differentiation differentiation

\section{INTRODUCTION}

Streptomyces spp. are Gram-positive soil bacteria that have been the focus of much study because of two particular characteristics. They undergo a complex cycle of morphological differentiation that culminates in the production of spores on the tips of aerial hyphae and they produce a host of secondary metabolites, many of which are medically important antibiotics. Among morphological mutants of the genetically well-characterized strain Streptomyces coelicolor A3(2) are the aerial-myceliumdeficient bld (for bald) mutants, many of which are also blocked in antibiotic production. The study of such pleiotropic mutants holds promise for a greater understanding of global regulatory signals that coordinately control the two very different aspects of differentiation. The best-characterized of the bld genes, bld $A$, encodes a leucyl tRNA (Lawlor et al., 1987) that recognizes the UUA codon, which is rare in Streptomyces mRNA because of the very high $\mathrm{G}+\mathrm{C}$ (approx. $73 \mathrm{~mol} \%$ ) content of their DNA. The isolation of pleiotropic mutants blocked in the expression of a rare tRNA, together with the observation that the rare codon is confined largely to genes that would be expressed only late in growth, such as antibiotic resistance and regulatory genes, has led to the suggestion that the bld $A$ gene might represent a translational regulatory mechanism controlling some antibiotic production and sporulation genes (Leskiw et al., 1991). More recently, it has been shown, at least under some

The GenBank accession number for the nucleotide sequence reported in this paper is U20761. growth conditions and for at least two Streptomyces spp. (Leskiw et al., 1993; N. K. Trepanier \& B. K. Leskiw, unpublished), that the bld $A$-encoded tRNA accumulates late in growth at a time that would be consistent with a regulatory role in morphological differentiation and antibiotic production.

We have been studying possible interconnections between other $b l d$ genes and expression of the bld $A$-encoded tRNA in an effort to determine if any of the bld genes are part of a regulatory pathway controlling bld $A$ expression. To date little is known about other bld gene products: bldD and bldG have been cloned (B. K. Leskiw \& R. Passantino, unpublished) but remain uncharacterized; and cloning and sequencing of $b l d B$ has failed to shed any light on a possible role in differentiation (Harasym et al., 1990). We have tested many of the available classes of bld mutants for bld $A$ expression, and based on the severely reduced bld $A$ expression in the bldI mutant of $S$. coelicolor, suggest that bldI is required either directly or indirectly for bld $A$ expression or function.

\section{METHODS}

Chemicals and enzymes. All electrophoresis chemicals were ultrapure grade. Taq DNA polymerase, and DNA-modifying enzymes, were from Boehringer Mannheim. Deep Vent DNA polymerase was from New England Biolabs. Nitrocefin was from Oxoid Unipath, thiostrepton was a gift from S. Lucania, Bristol-Myers Squibb, and viomycin was provided by Pfizer. All other chemicals were of reagent grade.

Bacterial strains, culture conditions and transformation procedures. $S$. coelicolor strains were J1501 [bis $A 1$ ura $A 1$ str $A 1$ 
pgl, SCP1- SCP2- ${ }^{-}$Chater et al. (1982)] and its derivatives J1681 [ $\triangle$ bld A; Leskiw et al. (1993)], C103 [bldG103; Champness (1988)], C181 [bldH181; Champness (1988), provided by W. Champness] and C249 [bld-249, proposed to be a bldI mutant (Champness 1988); Harasym et al. (1990), provided by W. Champness] shown here to be a bldB bld $A$ double mutant; J650 [cysD18 mtbB2 agaA1, NF SCP2*; Merrick (1976)] and its derivatives $\mathrm{J} 660[\mathrm{mthB} 2$ cysD18 aga $A 1$ bldC18, NF SCP2*; Merrick (1976)], J669 [mthB2 cysD18 aga A1 bldB43, NF SCP2*; Merrick (1976)] and J703 [mtbB2 aga $A 1$ bld-28, NF SCP2*; Harasym et al. (1990), provided by J. Piret]; the J1508 derivative HU66 [bis $A 1$ ura $A 1$ str $A 1$ pgl bldD 53, NF SCP2*; Willey et al. (1993), provided by J. Willey]; and 166 [bisD3 phe $A 1$ str $A 1$ bldF, SCP1* SCP2*; Passantino et al. (1991)]. All strains were obtained from K. Chater (John Innes Centre, Norwich, UK) unless otherwise indicated. Yeast extract/malt extract (YEME) medium (Hopwood et al., 1985) was used for Streptomyces liquid culture. Protoplasting and transformation of Streptomyces strains were as described by Hopwood et al. (1985). For RNA isolation, Streptomyces cultures were grown on cellophane discs (Leskiw et al., 1993; Soliveri et al., 1992) on the surface of R2YE agar (Hopwood et al., 1985).

Phage and plasmid vectors. The Streptomyces plasmids $\mathrm{pIJ} 584$ and pAU2 were high-copy-number derivatives of pIJ486 (Ward et al., 1986) containing bld $A$ and $a m p C$, respectively (Leskiw et al., 1993). Streptomyces plasmids were maintained by selection for thiostrepton resistance $\left[10 \mu \mathrm{g} \mathrm{ml}^{-1}\right.$; Hopwood et al. (1985)]. KC628 was a $\phi \mathrm{C} 31$ derivative carrying bldB (Harasym et al., 1990). Conditions for phage propagation were as described by Hopwood et al. (1985); phage integration to produce lysogens was scored as described by Piret \& Chater (1985) by selecting for resistance to viomycin $\left(20 \mu \mathrm{g} \mathrm{ml}^{-1}\right)$.

Preparation of cell extracts, and detection and assay of $\boldsymbol{\beta}$ lactamase activity. Cell extracts were prepared from mycelium scraped from the surface of cellophane discs as previously described (Leskiw et al., 1993). $\beta$-Lactamase activity was detected qualitatively by spraying colonies with the chromogenic cephalosporin nitrocefin $\left(0.5 \mathrm{mg} \mathrm{ml}^{-1}\right)$ as a substrate, and quantitatively by spectrophotometric assay as described previously (Leskiw et al., 1993). Specific $\beta$-lactamase activity is expressed as $\mathrm{mU}$ ( $\mathrm{mg}$ protein $)^{-1}$.

DNA procedures. Standard procedures were used for both Escherichia coli (Sambrook et al., 1989) and Streptomyces (Hopwood et al., 1985). Chromosomal DNA was isolated from Streptomyces strains using procedure 1 of Hopwood et al. (1985).

Sequence analysis of b/dA genes from Streptomyces strains. The template for sequencing was generated by PCR amplification of a $297 \mathrm{bp}$ fragment containing the bld $A$ tRNA region. The template for PCR was chromosomal DNA $(1 \mu \mathrm{g})$ from $S$. coelicolor J1501, J703 or C249, and the primers were a 28-mer synthetic oligonucleotide (5'-CATGGATCCACCCGGTAACTGATGCACC-3') corresponding to a region upstream of the bld $A$ promoter, and an 18-mer synthetic oligonucleotide $\left(5^{\prime}\right.$ GCCGCTGAGTCGCAACCA-3') corresponding to a sequence just downstream of the $\mathrm{SacII}$ site located $61 \mathrm{bp}$ from the end of the tRNA. Two independent PCR reactions were performed. One reaction employed Taq polymerase (Boehringer Mannheim) while the other employed the proofreading enzyme, Deep Vent DNA polymerase (New England Biolabs). Both reactions were carried out according to the manufacturers' specifications and the amplified bands were purified from a $5 \%$ $(\mathrm{w} / \mathrm{v})$ polyacrylamide gel by the crush and soak method (Sambrook et al., 1989). PCR products from both reactions were then directly sequenced using an ABI model 373A automated system (Department of Biological Sciences Sequencing Service, University of Alberta).
RNA manipulations. RNA was isolated at various intervals from mycelium grown on cellophane discs on the surface of R2YE agar, and Northern analysis was performed as previously described (Leskiw et al., 1993) except that $10 \mu \mathrm{g}$ of each total RNA sample was loaded per lane. In all cases the membranes were first hybridized to an end-labelled oligonucleotide, $5^{\prime}$ CCAGGGACTCGAACCCCG, complementary to the lys $T \alpha$ tRNA (Sedlmeier \& Schmieger, 1990), and then after stripping were hybridized to an end-labelled oligonucleotide, $5^{\prime}$ T'TAAGCTCGCCGTGTCT, complementary to the bld $A$ tRNA (Leskiw et al., 1993). In some cases RNA accumulation was quantified by densitometry of the exposed X-ray films with a BioImage XRS 3cx scanner.

The contribution of plasmid copy number variations to tRNA accumulation was determined after isolation of total DNA and RNA from the same cultures. Total nucleic acid was extracted and precipitated by the method of Kirby et al. (1967) as described by Hopwood et al. (1985), and then either DNA or RNA was selectively purified from half of the sample after treatment with RNase $\left(40 \mu \mathrm{g} \mathrm{m} \mathrm{ml}^{-1}\right.$, Sigma) or DNase

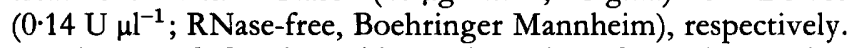
Northern and Southern blots of RNA and DNA samples, respectively, were then probed as described above.

Dot-blot analysis of ampC transcripts. Total RNA (10 $\mu \mathrm{g})$ was denatured as described by Ward et al. (1986), and applied to a Hybond-N nylon filter with a dot-blot apparatus. Hybridization was performed as previously described (Ward et al., 1986); the probe was a 410 bp HindIII-XhoI fragment internal to $a m p C$, and was labelled with $\left[\alpha^{32} \mathrm{P}\right] \mathrm{dATP}$ by the random primer method. All samples were treated with DNase-free RNase to ensure that the signals resulted from RNA transcripts.

\section{RESULTS}

\section{Some bld mutants are unable to translate UUA codons efficiently}

In an effort to determine if other classes of bld genes might play a role in regulating the presence of a functional bld $A$ tRNA, perhaps by involvement in transcription of the bld $A$ gene, $5^{\prime}$ or $3^{\prime}$ processing of the primary transcript, base modification, or charging by aminoacyl tRNA synthetase, the ability of $b l d B,-C,-D,-F,-G,-H$ and $-I$ mutants to translate UUA codons was tested. A highcopy-number plasmid derivative, pAU2, containing the ampC reporter gene (Leskiw et al., 1993) was introduced into each of the mutant strains by protoplast transformation. The ampC gene has seven TTA codons; its expression can be detected by spraying colonies with the chromogenic substrate nitrocefin, or it can be assayed spectrophotometrically using the same substrate. Although pIJ101-based vectors like pAU2 are incompatible with resident SCP1 plasmids (Schauer et al., 1991), as is the case in $\mathrm{SCP}^{+}$as well as NF strains (that have SCP1 integrated into the chromosome), pAU2 was maintained, albeit at a much lower copy number, in HU66, 166, and J650 and its derivatives by selection for thiostrepton resistance (data not shown). Preliminary screening of bld mutants involved qualitative assay for ampC $\beta$-lactamase activity. Transformants, including $\mathrm{J} 1501 / \mathrm{pAU} 2$ and $\mathrm{J} 1681 / \mathrm{pAU} 2$ as positive and negative controls respectively, were sprayed with nitrocefin and monitored for substrate cleavage by the appearance of a red/brown halo. All of the mutants tested were positive 
Table 1. Expression of ampC $\beta$-lactamase in extracts from cells of selected S. coelicolor strains

\begin{tabular}{|c|c|c|}
\hline Strain & $\begin{array}{c}\beta \text {-Lactamase activity } \\
\left(\mathrm{mU} \mathrm{mg} \mathrm{mg}^{-1}\right)\end{array}$ & Genotype \\
\hline J1501/pAU2 & $47 \pm 8 \cdot 6$ & bis $A 1$ ura $A 1$ str $A 1$ pgl, SCP1- SCP2- \\
\hline $\mathrm{J} 1681 / \mathrm{pAU} 2$ & $0 \cdot 11 \pm 0 \cdot 01$ & bis $A 1$ ura $A 1$ str $A 1$ pgl $\triangle b l d A, \mathrm{SCP} 1^{-} \mathrm{SCP} 2^{-}$ \\
\hline $\mathrm{J} 650 / \mathrm{pAU} 2$ & $17 \cdot 3 \pm 1 \cdot 6$ & $c y s D 18 m t b B 2$ aga $A 1$, NF SCP2* \\
\hline $\mathrm{J} 703 / \mathrm{pAU} 2$ & $0.86 \pm 0.24$ & $m t h B 2$ aga $A 1$ bld-28, NF SCP2* \\
\hline C249/pAU2 & $0 \cdot 11 \pm 0 \cdot 01$ & bis $A 1$ ura $A 1$ str $A 1$ pgl bld-249, SCP1- SCP2- \\
\hline
\end{tabular}

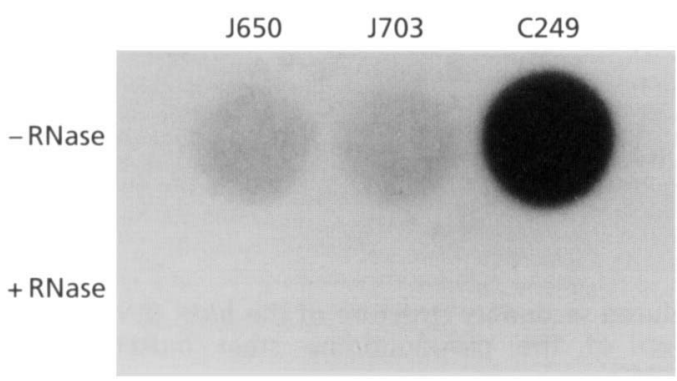

Fig. 1. Dot-blot analysis of ampC transcripts in RNA samples $(10 \mu \mathrm{g})$ isolated from the b/dl mutant $\mathrm{J703}$, and its parent strain strain J650. Controls included RNA isolated from the SCP1strain C249, and RNA samples treated with DNase-free RNase.

with the exception of $\mathrm{C} 249$ and $\mathrm{J} 703$, both reported to be bldI mutants, which showed no and severely reduced ampC expression, respectively. To rule out the possibility that reduced expression resulted from a change in the plasmid DNA, pAU2 was isolated from both J703 and C249 and used to transform J1501. In neither case was any defect seen in the expression of $a m p C$. The ampC-specified $\beta$-lactamase was then assayed quantitatively in cell extracts of C249 and J703 and their respective parent strains (Table 1). As with the qualitative assay, J1681/pAU2, the bld $A$ deletion mutant containing pAU2, served as a negative control. Two independent transformants were tested, and to account for differences in growth rates of the strains, mycelia were harvested at three times (between 30 and $72 \mathrm{~h}$ ); the highest activities are reported. Activities shown in Table 1 are means of values in duplicate extracts from the two independent transformants. As with the qualitative assay, C249 and J703 showed strikingly reduced $\beta$-lactamase activity. To show that the reduced expression of $a m p C$ did not result from a defect in ampC transcription, RNA isolated from C249, J703 and J650 was subjected to dot-blot analysis with an internal fragment of $a m p C$ as probe. The loss of signal after samples were digested with DNase-free RNase verified that the signals came from hybridization to RNA transcripts. RNA samples from both J650 and J703 showed identical levels of ampC transcripts, and, as expected due to the higher copy number of pAU2 in SCP1 ${ }^{-}$strains, C249 showed abundant ampC transcripts (Fig. 1). These results suggest that the reduced $\beta$-lactamase activity seen in C249 and J703 is not attributable to a lack

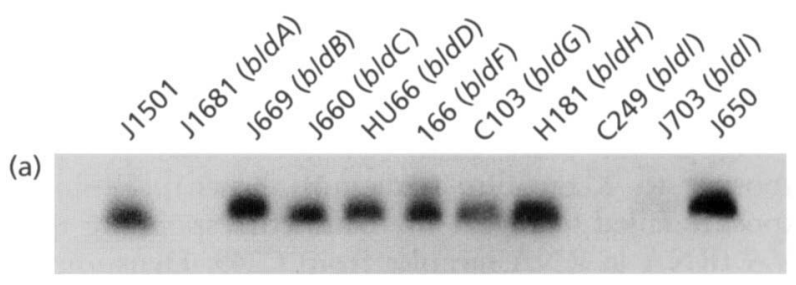

(b)

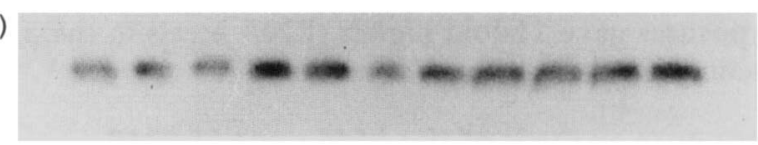

Fig. 2. (a) Northern analysis of the b/dA tRNA in RNA samples $(10 \mu \mathrm{g})$ isolated from surface-grown $S$. coelicolor b/dB, $-C,-D,-F$, $-G,-H$, and $-I$ mutants. Controls included parent strains from which the mutants were derived (see Methods) and a b/dA deletion mutant, $S$. coelicolor J1681. (b) The lysT $\alpha$ tRNA was probed as a control for RNA loading levels. RNA was isolated at $48 \mathrm{~h}$ post-inoculation. Probes were end-labelled oligonucleotides complementary to the b/dA and $/ y s T \alpha$ tRNAs (see Methods).

of $\operatorname{ampC}$ transcription, and furthermore that these strains have mutations that affect the availability of translationproficient bld $A$ tRNA.

\section{Expression of b/dA-encoded tRNA in b/dl mutants}

Inability to express $a m p C$ efficiently due to limited translation of its UUA codons could result either from a failure to transcribe the bld $A$ gene or from a defect in processing or modification of the bld $A$ transcript. To investigate the possibility that the level of the bld $A$ tRNA might be reduced in C249 and J703, total RNA samples isolated from $48 \mathrm{~h}$ surface cultures of all of the bld mutant and parent strains described above were subjected to Northern analysis. The probes were end-labelled oligonucleotides complementary to the bld $A$ tRNA and, as a control for RNA loading levels, lysT $\alpha$ tRNA sequences. The Northern analysis was performed on RNA isolated from at least two independent transformants; representative results are shown in Fig. 2. As expected, the bld strains with no impairment in ampC activity had bld $A$ tRNA levels comparable to those in the parent strains. However, the bld $A$ tRNA could not be detected in C249, and was present at very low levels in J703 [the faint band 


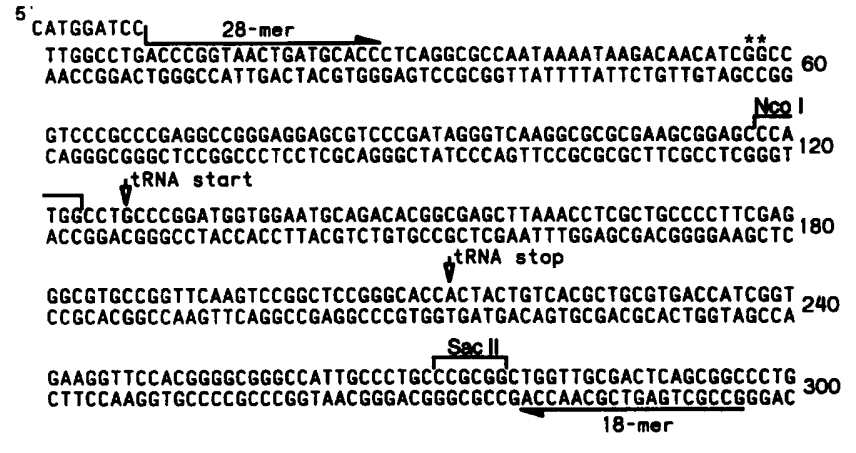

Fig. 3. DNA sequence of the b/dA region. Primers used for PCR amplification of a 297 bp fragment are shown. **, the previously determined location of the bldA transcription start point (Leskiw et al., 1993).

in Fig. 2 was more easily visualized after prolonged exposure to X-ray film (data not shown)]. Prolonged exposure failed to reveal any band corresponding to the bld $A$ tRNA in RNA samples from C249. Densitometric scanning of two independent autoradiograms from long exposures gave 11-fold higher tRNA levels in the parent strain J650 than in the bldI mutant J703.

\section{Sequence analysis of the b/dA gene in C249}

The lack of any detectable tRNA, together with the complete absence of $a m p C$ activity, in C249 mirrored the pattern in the bld $A$ mutant, J1681, and contrasted with the low but detectable tRNA levels and ampC activity in J703. These observations suggested that C249 might carry a bld $A$ mutation. To investigate this possibility a fragment of DNA containing bld $A$ was amplified from chromosomal DNA of C249 by PCR, and sequenced directly using the same primers as were used for the amplification (Fig. 3; see Methods). Controls included PCR-amplified bld $A$ fragments from both $\mathrm{J1501}\left(\right.$ bld $A^{+}$) and J703. PCR amplifications were performed independently with Taq polymerase and with Deep Vent polymerase and both of the resulting amplified fragments were sequenced. The sequencing (Fig. 4) revealed a mutation changing the pseudouridine $(\psi)$ stem of the bld $A$ tRNA in C249. No mutations were found within the amplified sequence from $\mathrm{J} 703$.

\section{Complementation of the bld phenotype in C249}

The finding of a bld $A$ mutation in C249, together with the fact that the bld-249 mutation mapped to the bldB region (Champness, 1988), suggested that $\mathrm{C} 249$ might represent a bld $A$ bldB double mutant. To verify this, C249 containing $\mathrm{pIJ} 584$, a high-copy-number plasmid derivative of bld $A$ (Leskiw et al., 1993), was lysogenized with a $\phi \mathrm{C} 31$ phage vector, KC628, carrying the bldB gene. Lysogens resistant to both viomycin and thiostrepton, the selectable markers present on the phage and the plasmid, respectively, were isolated. All lysogens expressing both resistance markers were able to sporulate and produce the pigmented antibiotics characteristic of wild-type $S$. coelicolor, confirming that C249 represents a double mutant.

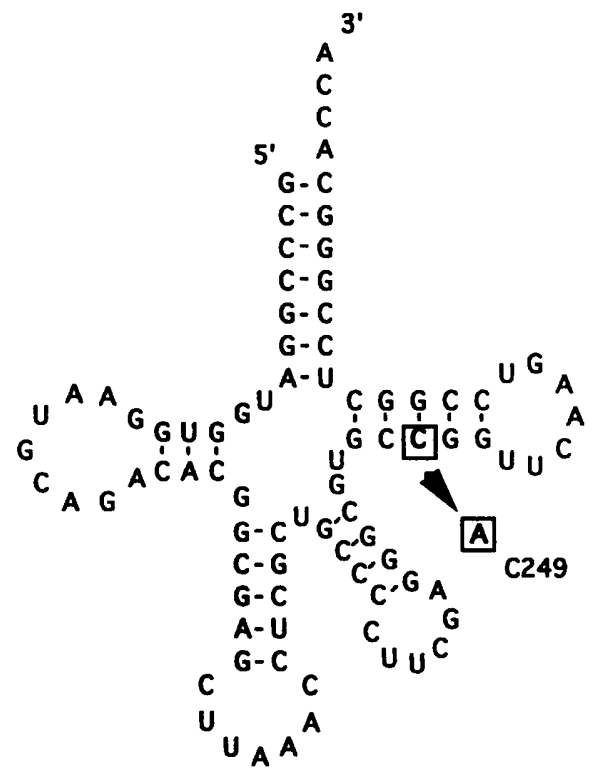

Fig. 4. Deduced secondary structure of the bldA tRNA showing the position of the pseudouridine stem mutation in $S$. coelicolor C249.

\section{Introduction of a high-copy-number plasmid containing b/dA into $\mathrm{J703}$}

To rule out the possibility that reduced bld $A$ tRNA levels in $\mathrm{J} 703$ resulted from a mutation in the promoter region of the bld $A$ gene (the location of the sequencing primers shown in Fig. 3 did not allow the entire promoter region to be sequenced), the bld $A$ gene from strain $\mathrm{J} 1501\left(\right.$ bld $\left.A^{+}\right)$ was introduced into J650 and J703 on the high-copynumber plasmid derivative, $\mathrm{pIJ584}$. RNA was isolated at various times from two independent transformants of each strain and Northern analysis was performed as described above. Controls included J1501, J1681 and C249 containing pIJ584, as well as pAU2-containing transformants of those strains to show the single-copy expression levels for the bld $A$ tRNA. Typical results are shown in Fig. 5. J703 showed reduced expression of the bld $A$ tRNA, even when the bld $A$ gene was present on a high-copy-number plasmid. Densitometric analysis of the signals from undiluted RNA samples taken at $53 \mathrm{~h}$ (from a time-course of samples isolated at 20,29 and $53 \mathrm{~h}$ ) and from $72 \mathrm{~h}$ cultures (from a time-course of samples isolated at 24,48 and $72 \mathrm{~h}$ ) of $\mathrm{J} 703 / \mathrm{pIJ} 584$, and serially diluted RNA samples from J650/pIJ584, isolated from the same time-courses, showed 6.5- and 6.9-fold increases in the bld $A$ tRNA levels in J650 as compared to J703 (data not shown). Comparisons of densitometry values for signals from all of the time-course points are not reported because the signals were too low to be detected from the J703 samples. To verify the structural stability of the plasmid, pIJ584 was also isolated from both J703 and C249 and transformed into the bld $A$ deletion mutant J1681. In both cases high levels of tRNA were seen in the J1681 transformants (Fig. 5). To rule out plasmid copy number differences as a possible explanation for the differences in bld $A$ tRNA expression, total DNA samples from the cultures used for RNA isolation were also subjected to 


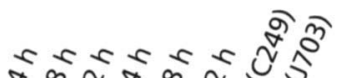
$\alpha \infty \approx \alpha \infty \approx$

(a)

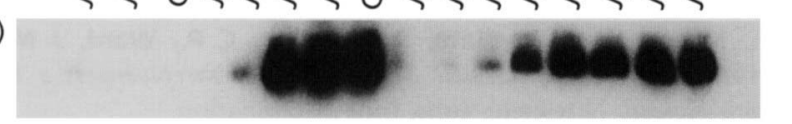

(b)

Fig. 5. (a) Northern analysis of the b/dA tRNA in RNA samples $(10 \mu \mathrm{g})$ isolated from surface-grown $S$. coelicolor bldl mutants containing b/dA on a high-copy-number plasmid (plJ584). Controls included RNA isolated from: parent strains from which the mutants were derived (see Methods); the b/dA deletion mutant S. coelicolor J1681; all parent and mutant strains with the same high-copy-number replicon containing the ampC gene (pAU2) in place of pIJ584; and in the two right-hand lanes, strain $\mathrm{J1681}$ after transformation with plasmid plJ584 from $S$. coelicolor C249 and $\mathrm{J703}$ (strains from which the plasmids were isolated for transformation into $J 1681$ are shown in parentheses). (b) The lysT $\alpha$ tRNA was probed as a control for RNA loading levels. RNA was isolated at $48 \mathrm{~h}$ post-inoculation unless otherwise indicated. Probes were end-labelled oligonucleotides complementary to the b/dA and lysTa tRNAs (see Methods). (a)

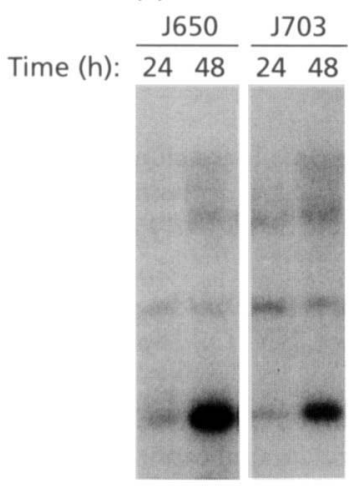

(b)

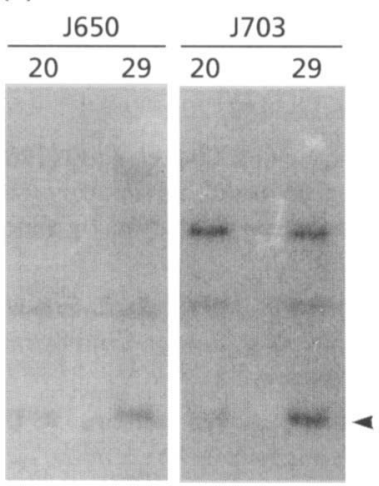

Fig. 6. Southern analysis for comparison of plJ584 (a high-copynumber plasmid derivative carrying the bldA gene) levels in total DNA samples isolated from the the b/d/ mutant, J703, and its parent strain, J650. DNA samples isolated at $24 \mathrm{~h}$ and $48 \mathrm{~h}$ are shown in (a); DNA samples isolated from a separate timecourse at $20 \mathrm{~h}$ and $29 \mathrm{~h}$ are shown in (b). The probe was the b/dA-specific, end-labelled oligonucleotide also used to detect the bldA tRNA in Figs 2 and 5. The arrowhead indicates the CCC form of the plasmid DNA. Slower-migrating bands most likely represent relaxed circle and linear forms of the plasmid.

Southern analysis. Both strains gave similar results, with markedly higher copy numbers at later times (Fig. 6). Densitometry of the bands corresponding to CCC plasmid DNA in Fig. 6(a) showed no difference between the two strains in plasmid levels in the $24 \mathrm{~h}$ DNA samples, and only a $1 \cdot 4$-fold difference at $48 \mathrm{~h}$. Likewise the samples isolated at 20 and $29 \mathrm{~h}$ (Fig. 6b) showed virtually no differences in plasmid levels between strains. Additional, slower-migrating bands, most likely corresponding to linear and relaxed circle forms of the plasmid, are also visible on the blot; however, virtually no differences were seen in the levels of these bands between the strains.

\section{DISCUSSION}

Previous studies on $b l d A$ transcription indicated that the gene was transcribed from a promoter that was active at all times during growth, and that the $5^{\prime}$ processed form of the tRNA showed increased accumulation only in older cultures (Leskiw et al., 1993). These results suggested that the temporal appearance of the mature form of the tRNA was not principally regulated at the transcriptional level, but rather at the level of processing of the primary transcript to give a mature $5^{\prime}$ end. To further our understanding of regulation of the appearance of a translation-proficient form of the bld $A$-encoded tRNA, we undertook a study of the expression of the bld $A$ gene in other classes of bld mutants of $S$. coelicolor.

When expression of the TTA-containing reporter gene ampC was assayed in cell extracts prepared from $b l d B,-C$, $-D,-F-G,-H$ and $-I$ mutants, only bldI mutants showed a defect in ampC activity. Of the two proposed bldI strains, C249 proved to be a bld $A$ bld $B$ double mutant, and will not be discussed further. A defect in secretion of the ampC-specified $\beta$-lactamase could not account for the reduced activity in the bldI mutant J703 because the enzyme is not secreted in Streptomyces spp. (Häggström \& Jaurin, 1985). Furthermore, the reduced ampC activity was not attributable to a defect in ampC transcription, suggesting that the bldI mutant is impaired in expression of the bld $A$ tRNA. Indeed, when Northern analysis was used to assess mature bld $A$ tRNA levels, J703 showed much lower tRNA levels than its parent strain J650, and the strikingly reduced tRNA levels were also seen when a high-copy-number plasmid containing bld $A$ was introduced into J703. Since the bld $A$ tRNA is required for morphological differentiation in $S$. coelicolor, these results suggest that the bld phenotype and the bld $A$ tRNA deficiency in $\mathrm{J} 703$ are aspects of the same mutation. However, it is clear that the bldI mutation has manifestations separate from those concerned with bld $A \mathrm{tRNA}$ expression, since introducing the high-copy-number derivative of bld $A$ into $\mathrm{J} 703$ increased the tRNA to a level similar to that in the parent strain J650, but this increase did not abolish the defect in aerial mycelium formation. (It should be noted that our experiments did not address the possibility that the $b l d I$ gene product plays a role in modification of the tRNA into a functional, translationproficient form. Although this explanation would account for the inability to restore aerial mycelium after overexpression on a high-copy-number plasmid, it would not account for the reduced levels of the processed, $87 \mathrm{nt}$ form of the tRNA in J703 relative to the parent J650.) That $b l d I$ may have effects unrelated to bld $A$ expression is not entirely surprising in view of the differences in phenotypes between these bld mutants. bld $A$ mutants show carbon-source-dependent restoration of aerial my- 
celium formation, whereas the bldI mutant phenotype is unaffected by the carbon source (Harasym et al., 1990).

The low level of mature bld $A$ tRNA expression in $\mathrm{J} 703$, even under conditions where bld $A$ was present on a highcopy-number plasmid, suggest that the bldI mutant may lack a positive transcriptional activator that is required for maximal bld $A$ expression. The bldI gene product might itself interact directly with the bld $A$ promoter region, or it could be required for expression of an as yet unidentified transcription factor. Whether the effect is direct or indirect, the requirement of a functional bldI product for maximal bld $A$ expression suggests an additional level in the regulatory cascade controlling some antibiotic production and sporulation genes. Since a low level of the bld $A$ tRNA is expressed in J703, the increased accumulation of the tRNA in older cultures of wild-type $S$. coelicolor may well represent transcription from a second, perhaps bldI-dependent, promoter. Although previous S1 nuclease analysis of bld $A$ transcripts failed to reveal a second promoter, an unexplained series of bands 21-23 nt upstream of the beginning of the tRNA was present in those analyses (Leskiw et al., 1993) and it is conceivable that they represent transcripts initiating at a separate promoter. Alternatively, as is the case with most $t \mathrm{RNAs}$ in E. coli, transcripts arising from a second promoter may be processed so quickly that precursor tRNA species are not seen [except, for example, in the case where the tRNA has mutations which alter the secondary or tertiary structure (King \& Schlessinger, 1987)]. This very rapid processing to the mature tRNA appears to be the case for bld $A$ transcripts produced late in growth (Leskiw et al., 1993). As an alternative to an effect of bldI on bld $A$ transcription, the reduced tRNA levels in the bldI mutant might be explained by an inability to process the primary transcript to the mature $5^{\prime}$ processed form. Since bands corresponding to tRNA precursors were not detected by Northern analysis in this or in previous studies (Leskiw $e t$ al., 1993), this possibility has not been ruled out. These questions will most easily be answered by determining the nature of the bldI gene product.

\section{ACKNOWLEDGEMENTS}

This work was supported by the Alberta Heritage Foundation for Medical Research, and the Natural Sciences and Engineering Research Council of Canada. We thank Kevin Wenzel and Leigh Ann Giebelhaus for technical assistance, Wendy Champness, Joanne Willey, Keith Chater and Jacqueline Piret for donation of strains, Wendy Champness and Jacqueline Piret for helpful discussions, and Keith Chater for helpful comments on the manuscript.

\section{REFERENCES}

Champness, W. C. (1988). New loci required for Streptomyces coelicolor morphological and physiological differentiation. $J$ Bacteriol 170, 1168-1174.

Chater, K. F., Bruton, C. J., King, A. A. \& Suarez, J. E. (1982). The expression of Streptomyces and Escherichia coli drug resistance determinants cloned into the Streptomyces phage $\phi \mathrm{C} 31$. Gene 19, 21-32.

Hăggström, B. \& Jaurin, B. (1985). Secretion in Streptomyces lividans.
In Proceedings of the Sixth International Symposium on the Biology of Actinomycetes, Debrecen, Hungary, Abstract M134.

Harasym, M., Zhang, L.-H., Chater, K. \& Piret, J. (1990). The Streptomyces coelicolor A3(2) bldB region contains at least two genes involved in morphological development. J Gen Microbiol 136, 1543-1550.

Hopwood, D. A., Bibb, M. J., Chater, K. F., Kieser, T., Bruton, C. J., Kieser, H. M., Lydiate, D. J., Smith, C. P., Ward, J. M. \& Schrempf, H. (1985). Genetic Manipulation of Streptomyces: a Laboratory Manual. Norwich: John Innes Foundation.

King, T. C. \& Schlessinger, D. (1987). Processing of RNA transcripts. In Escherichia coli and Salmonella typhimurium: Cellular and Molecular Biology, pp. 703-718. Edited by F. C. Neidhardt, J. L. Ingraham, K. Brooks Low, B. Magasanik, M. Schaechter \& H. E. Umbarger. Washington, DC: American Society for Microbiology.

Kirby, K. S., Fox-Carter, E. \& Guest, M. (1967). Isolation of deoxyribonucleic acid and ribosomal ribonucleic acid from bacteria. Biochem J 104, 258-262.

Lawlor, E. J., Baylis, H. A. \& Chater, K. F. (1987). Pleiotropic morphological and antibiotic deficiencies result from mutations in a gene encoding a tRNA-like product in Streptomyces coelicolor A3(2). Genes \& Dev 1, 1305-1310.

Leskiw, B. K., Bibb, M. J. \& Chater, K. F. (1991). The use of a rare codon specifically during development? Mol Microbiol 5, 2861-2867.

Leskiw, B. K., Mah, R., Lawlor, E. J. \& Chater, K. F. (1993). Accumulation of bld $A$-specified tRNA is temporally regulated in Streptomyces coelicolor A3(2). J. Bacteriol 175, 1995-2005.

Merrick, M. J. (1976). A morphological and genetic mapping study of bald colony mutants of Streptomyces coelicolor. J Gen Microbiol 96, 299-315.

Passantino, R., Puglia, A. M. \& Chater, K. F. (1991). Additional copies of the actII regulatory gene induce actinorhodin production in pleiotropic bld mutants of Streptomyces coelicolor A3(2). J Gen Microbiol 137, 2059-2064.

Piret, J. M. \& Chater, K. F. (1985). Phage-mediated cloning of bld $A$, a region involved in Streptomyces coelicolor morphological development and its analysis by genetic complementation.J Bacteriol 163, 965-972.

Sambrook, J., Fritsch, E. F. \& Maniatis, T. (1989). Molecular Cloning: a Laboratory Manual. Cold Spring Harbor, NY: Cold Spring Harbor Laboratory.

Schauer, A. T., Nelson, A. D. \& Daniel, J. B. (1991). Tn4563 transposition in Streptomyces coelicolor and its application to isolation of new morphological mutants. J Bacteriol 173, 5060-5067.

Sedlmeier, R. \& Schmieger, H. (1990). Nucleotide sequences of tRNA genes in Streptomyces lividans 66. Nucleic Acids Res 18, 4027.

Soliveri, J., Brown, K. L., Buttner, M. J. \& Chater, K. F. (1992). Two promoters for the whiB sporulation gene of Streptomyces coelicolor A3(2) and their activities in relation to development. J Bacteriol 174, 6215-6220.

Ward, J. M., Janssen, G. R., Kieser, T., Bibb, M. J., Buttner, M. J. \& Bibb, M. J. (1986). Construction and characterisation of a series of multi-copy promoter-probe plasmid vectors for Streptomyces using the aminoglycoside phosphotransferase gene from $\operatorname{Tn} 5$ as indicator. Mol \& Gen Genet 203, 468-478.

Willey, J., Schwedock, J. \& Losick, R. (1993). Multiple extracellular signals govern the production of a morphogenetic protein involved in aerial mycelium formation by Streptomyces coelicolor. Genes \& Dev 7, 895-903.

Received 24 January 1995; revised 7 April 1995; accepted 24 April 1995. 